\title{
Survival analysis and prognostic factors of palliative radiotherapy in patients with metastatic colorectal cancer: a propensity score analysis
}

\author{
$\mathrm{Li} \mathrm{Ba}^{1 \# \wedge}$, Qingrui Wang ${ }^{2 \#}$, Haihong Wang ${ }^{3}$, Lisheng Zhu $^{3}$, Tao Zhang ${ }^{3}$, Jinghua Ren ${ }^{3}$, Zhenyu Lin ${ }^{3}$ \\ ${ }^{1}$ Department of Ultrasound, Union Hospital, Tongji Medical College, Huazhong University of Science and Technology, Wuhan, China; ${ }^{2}$ Department \\ of Oncology, The Sixth Hospital of Wuhan (Affiliated Hospital of Jianghan University), Jianghan University, Wuhan, China; ${ }^{3}$ Cancer Center, Union \\ Hospital, Tongji Medical College, Huazhong University of Science and Technology, Wuhan, China \\ Contributions: (I) Conception and design: L Ba, Z Lin; (II) Administrative support: T Zhang, J Ren; (III) Provision of study materials or patients: \\ Q Wang, T Zhang; (IV) Collection and assembly of data: H Wang, L Zhu; (V) Data analysis and interpretation: H Wang, L Zhu; (VI) Manuscript \\ writing: All authors; (VII) Final approval of manuscript: All authors. \\ \#These authors contributed equally to this work. \\ Correspondence to: Zhenyu Lin, MD. Cancer Center, Union Hospital, Tongji Medical College, Huazhong University of Science and Technology, \\ Wuhan 430022, China. Email: whxhlzy@hust.edu.cn.
}

Background: Radiation therapy (RT) is known to have beneficial effects on the palliative treatment of patients with advanced cancer. However, valid data on this treatment method are limited, especially for patients with metastatic colorectal cancer (mCRC). This study aimed to identify prognostic factors and investigate the outcomes of mCRC patients who received palliative RT.

Methods: A total of $488 \mathrm{mCRC}$ patients who underwent systemic therapy with or without palliative RT between 2014 and 2019 were included in the study. Of the 488 patients, 155 received systemic treatment combined with palliative RT (RT group), while 333 were only administered systemic treatment (non-RT group). Propensity score matching (PSM) was conducted to eliminate possible bias, and overall survival (OS) was calculated using the Kaplan-Meier (KM) method. A log-rank test was used to compare the survival outcomes of each group, and a multivariate analysis was conducted using a Cox proportional hazards model.

Results: The RT group had a higher OS than that of the non-RT group ( $\mathrm{P}=0.001)$. After PSM, the median OS of the RT group was 50.8 months, and for the non-RT group it was 32.2 months $(\mathrm{P}=0.003)$. Subgroup analysis revealed that RT had a better effect on the OS of patients who had synchronous metastasis, or who didn't receive targeted therapy or local treatment (including surgery, ablation, and intervention). Multivariate analysis of the whole cohort showed that palliative RT was associated with improved OS. Moreover, multivariate analysis of the RT group showed that systemic therapy before RT, and the site of RT was in the liver and lung, were independent prognostic factors affecting survival time.

Conclusions: We demonstrated that systemic treatment followed by palliative RT led to a better OS for mCRC patients. This combination method can therefore be seen as a suitable treatment approach for patients with mCRC.

Keywords: Metastatic colorectal cancer (mCRC); palliative radiotherapy; propensity score matching (PSM); survival analysis

Submitted Aug 05, 2021. Accepted for publication Sep 28, 2021.

doi: 10.21037/jgo-21-540

View this article at: https://dx.doi.org/10.21037/jgo-21-540

^ ORCID: 0000-0002-7959-0634. 


\section{Introduction}

Colorectal cancer (CRC) is one of the most common cancers in both men and women, with approximately 140,000 new cases identified annually (1). About $20-25 \%$ of CRC patients have distant metastasis at the time of diagnosis, and over half of CRC patients may develop metastasis during disease progression (2). According to the National Comprehensive Cancer Network (NCCN) guidelines for CRC, chemotherapy combined with targeted agents is the main treatment option for CRC patients and the median overall survival (OS) is now approximately 30 months (3-6). However, in recent years, the efficacy of chemotherapy appears to have plateaued.

Radiation therapy (RT) is one of the most effective treatments for malignant tumors. About $70 \%$ of patients with malignant tumors will require RT during their course of illness. For patients with advanced cancer, palliative RT can relieve cancer symptoms, improve quality of life, and even prolong survival. In recent years, a variety of studies have suggested that RT combined with systemic therapy for advanced tumor patients can significantly improve survival (7-9). Recently, a multicenter, randomized, controlled phase II study showed how combining local RT with systemic therapy for treating patients with oligometastatic nonsmall cell lung cancer improved the OS (the data shows OS reached 41.2 months) (10). In another study, combining RT with neoadjuvant therapy for locally advanced rectal cancer also confirmed an improvement in OS. In addition, numerous other studies have shown that both longcourse concurrent chemoradiotherapy and short-course segmented radiotherapy, can reduce the local recurrence (LR) rate and improve disease-free survival (11-14). Given the lack of high-level evidence, however, RT as a neoadjuvant or adjuvant treatment for resectable colon cancer is not recommended under current guidelines. In advanced CRC, RT is mainly a palliative treatment that relieves symptoms. It remains controversial as to whether RT can improve prognosis and the OS of metastatic CRC (mCRC) patients. Although in recent years increasing evidence has suggested that some mCRC patients have benefited from palliative RT, the majority of these studies only included patients with oligometastatic CRC or rectal cancer. Furthermore, these patients were usually treated with stereotactic body RT (SBRT).

In the absence of randomized data on the subject, we conducted a retrospective study to investigate whether the combination of palliative RT and systemic therapy could improve the OS of patients, and to evaluate the potential prognostic factors of this treatment method. Propensity score matching (PSM) is a technique that used to balance the confounding factors. In this study, the control group subjects were matched with the study group subjects by means of the propensity score method. We present the following article in accordance with the STROBE reporting checklist (available at https://dx.doi. org/10.21037/jgo-21-540).

\section{Methods}

\section{Patients}

This retrospective study included 488 patients diagnosed with mCRC between December 2014 and October 2019 at the Cancer Center, Union Hospital, Tongji Medical College, Huazhong University of Science and Technology (Wuhan, China). The criteria for selecting patients were as follows: (I) diagnosed with mCRC histopathologically or cytologically; (II) age $\geq 18$ years and $\leq 80$ years; (III) no history of other cancer; (IV) information on survival time was available. The cohort was divided into two groups according to whether they underwent RT (RT group) or did not (non-RT group). Radiation to tumor was implemented by modern radiotherapeutic techniques [3D conformal RT (3DCRT) or intensity modulated RT (IMRT) or SBRT]. The timing of radiotherapy depends on the patient's condition. All procedures performed in this study involving human participants were in accordance with the Declaration of Helsinki (as revised in 2013). The study was approved by the ethics committee of the Wuhan Union Hospital. Because of the retrospective nature of the research, the requirement for informed consent was waived.

\section{PSM}

PSM is a method to reduce the selection bias in nonrandomized studies $(15,16)$. For our analysis, we created a matching dataset using the following baseline covariates as the matching factors: age, gender, surgery status, $T$ stage, $\mathrm{N}$ stage, time to metastasis, and KRAS status. The RT group and non-RT group were matched 1:1 using nearest neighbor matching, setting the caliper as 0.02 .

\section{Statistical analyses}

The primary concern of this study was a patient's OS, 
which was defined as the time from the date of diagnosis to the date of death (due to mCRC or any other cause). Comparison of clinical characteristics were analyzed using a Chi-square test. Survival curves for both outcomes were drawn using the Kaplan-Meier (KM) method, and a log-rank test was used to compare differences between the groups. Using the statistically significant variables obtained through univariate analysis, a Cox proportionalhazards model was then applied to perform the multivariate analyses. PSM was also used to adjust the potential impact of heterogeneity. $\mathrm{P}$ value less than $0.05(\mathrm{P}<0.05)$ was considered statistically significant. All statistical analyses were performed using IBM SPSS Statistics 25.0, and the survival curve was generated by $\mathrm{R}$ (version 3.6.1).

\section{Results}

\section{Patient characteristics}

A total of 488 patients newly diagnosed with mCRC met the eligible criteria for this study. One hundred and fiftyfive patients who received RT were classified as the RT group, while the other 333 patients were allocated to the non-RT group. The clinical and pathologic characteristics of all mCRC patients are summarized in Table 1. Patients with metachronous metastasis were most likely to receive RT $(58.1 \%$ vs. $29.1 \%, \mathrm{P}<0.01)$, while patients at stage T3 (47.7\% vs. $33.9 \%, \mathrm{P}<0.01)$, stage $\mathrm{N} 1(34.0 \%$ vs. $41.2 \%$, $\mathrm{P}<0.01)$, or who underwent surgery $(93 \%$ vs. $75.4 \%$, $\mathrm{P}<0.01$ ), were also more likely to receive RT. Considering the significant differences between the RT and non-RT group, we used PSM to balance the distribution of most demographic and clinical characteristics. In the PSM cohort, 144 well-balanced pairs were available for outcome comparison. After PSM, there were almost no statistically significant differences in baseline characteristics between the two groups, except for whether the patient received local RT treatment.

\section{The improved survival of $m C R C$ patients who underwent RT before PSM}

At the end of the follow-up time, the median OS of patients in the RT group was 50.8 months, and 29.2 months in the nonRT group. The 1- and 2-year OS rates of the RT and non-RT group were $73.5 \%$ vs. $63.4 \%$, and $39.4 \%$ vs. $19.2 \%$ ( $\mathrm{P}=0.001$, Figure $1 A$ ), respectively. These results suggested that $\mathrm{RT}$ was associated with a longer OS in patients with $\mathrm{mCRC}$.

\section{Survival analysis of the propensity score-matched cobort}

To eliminate the imbalance of clinicopathological features between the RT and non-RT groups, we used PSM to perform a 1:1 cohort analysis. In this propensity-matched cohort $(n=288)$, the median OS in the RT group was 50.8 months, while in the non-RT group it was 32.2 months. The 1- and 2-year OS rates in the RT group were $73.6 \%$ and $40.3 \%$, respectively, while in the nonRT group they were $61.1 \%$ and $22.9 \%$, respectively. Survival curves in the two groups are shown in Figure $1 B$. Additionally, we performed univariate and multivariate analyses to identify significant predictors of OS. The univariate analysis of all patients indicated that the following factors were significantly associated with OS: age, site of primary tumor, $\mathrm{N}$ stage, surgery of primary site, RT, and the local treatment of metastasis. Through the multivariate analysis, the site of primary tumor, surgery of primary site, local treatment of metastasis, and RT retained their significance (Table 2). The above results confirmed that the improved OS of the RT group was maintained in the propensity score-matched group.

\section{Exploratory subgroup analysis after PSM and according to the clinical evaluation of $R T$}

To identify which patients benefited from RT, we conducted a subgroup analysis according to whether the patients received targeted therapy, whether they received local treatment of metastasis, and the status of metastasis (simultaneous/metachronous). The results of the simultaneous metastasis subgroup showed that the OS in the RT group was significantly longer than that of the non-RT group ( $\mathrm{P}=0.001$, Figure $2 A)$. Nevertheless, there was no significant difference in the metachronous metastasis subgroup (Figure 2B). As shown in Figure 2C,2D, RT significantly improved the OS of patients who did not receive targeted therapy $(\mathrm{P}<0.001)$. In addition, we analyzed the role of $\mathrm{RT}$ in patients who did not receive local treatment and found it had more prognostic significance than for those who received local treatment $(\mathrm{P}<0.001)$. This is illustrated in Figure 2E, $2 F$.

\section{Prognostic factors predicting survival among patients treated with $R T$}

Multivariate Cox proportional-hazards regression analysis was used to predict survival among RT patients. These 
Table 1 Baseline characteristics of mCRC patients before and after PSM

\begin{tabular}{|c|c|c|c|c|c|c|}
\hline Variables & \multicolumn{3}{|c|}{ Overall sample $(n=488)$} & \multicolumn{3}{|c|}{ Propensity-matched sample $(n=288)$} \\
\hline Total & 155 & 333 & & 144 & 144 & \\
\hline Age (year) & & & 0.512 & & & 0.632 \\
\hline$\geq 65$ & $22(14.2)$ & $55(16.5)$ & & $22(15.3)$ & $25(17.4)$ & \\
\hline Gender & & & 0.516 & & & 0.549 \\
\hline Male & $94(60.6)$ & $192(57.7)$ & & $83(57.6)$ & $88(61.1)$ & \\
\hline Female & $61(39.4)$ & $141(42.3)$ & & $61(42.4)$ & $56(38.9)$ & \\
\hline Stage of diagnosis & & & $<0.001$ & & & 1.000 \\
\hline Site of primary tumor & & & 0.081 & & & 0.790 \\
\hline Left-colon & $45(29)$ & $112(33.6)$ & & $44(30.6)$ & $51(35.4)$ & \\
\hline Right-colon & $24(15.5)$ & $78(23.4)$ & & $24(16.7)$ & $28(19.4)$ & \\
\hline NA & $5(3.2)$ & $12(7.7)$ & & $5(3.4)$ & $5(3.4)$ & \\
\hline Histology & & & 0.617 & & & 0.745 \\
\hline Adenocarcinoma & 144 (92.9) & 297 (89.2) & & $133(92.4)$ & $130(90.3)$ & \\
\hline MAD & $9(5.8)$ & $23(6.9)$ & & $9(6.3)$ & $11(7.6)$ & \\
\hline SRC & $2(1.3)$ & $8(2.4)$ & & $2(1.4)$ & $2(1.4)$ & \\
\hline NA & $17(11)$ & $84(25.2)$ & & $17(11.7)$ & $14(9.7)$ & \\
\hline $\mathrm{N}$ & & & 0.007 & & & 0.803 \\
\hline No & 32 (20.6) & $56(16.8)$ & & $30(20.8)$ & 28 (19.4) & \\
\hline $\mathrm{N} 1$ & 48 (31.0) & 89 (26.7) & & $44(30.6)$ & $51(35.4)$ & \\
\hline N2 & $56(36.1)$ & $100(30.0)$ & & $53(36.8)$ & 48 (33.3) & \\
\hline NA & 19 (12.3) & $88(26.4)$ & & $17(11.8)$ & 17 (11.8) & \\
\hline Surgery $^{a}$ & & & $<0.001$ & & & 1.000 \\
\hline No surgery & $11(7.0)$ & $82(24.6)$ & & $11(7.6)$ & $11(7.6)$ & \\
\hline Surgery & $144(93.0)$ & $251(75.4)$ & & $133(92.4)$ & $133(92.4)$ & \\
\hline
\end{tabular}

Table 1 (continued) 
Table 1 (continued)

\begin{tabular}{|c|c|c|c|c|c|c|}
\hline Variables & \multicolumn{3}{|c|}{ Overall sample $(n=488)$} & \multicolumn{3}{|c|}{ Propensity-matched sample $(\mathrm{n}=288)$} \\
\hline Local treatment $^{\mathrm{b}}$ & & & 0.542 & & & 0.049 \\
\hline Yes & $46(29.7)$ & $108(32.4)$ & & $43(29.9)$ & $59(41.0)$ & \\
\hline No & $109(70.3)$ & $225(67.6)$ & & $101(70.1)$ & $85(59.0)$ & \\
\hline MT & $38(24.5)$ & $81(24.3)$ & & $35(24.3)$ & $38(26.4)$ & \\
\hline WT & $33(21.3)$ & $68(20.4)$ & & $27(18.8)$ & $30(20.8)$ & \\
\hline NA & $84(54.2)$ & $184(55.3)$ & & $82(56.9)$ & $76(52.8)$ & \\
\hline Molecular targeting & & & 0.630 & & & 0.631 \\
\hline
\end{tabular}

${ }^{\text {a }}$, surgery of primary site; ${ }^{b}$, local treatment of metastasis (including surgery, ablation, and intervention). mCRC, metastatic colorectal cancer; PSM, propensity score matching; MAD, mucinous adenocarcinoma; SRC, signet ring cell carcinoma; MT, mutation; WT, wild-type; NA, not available.

A

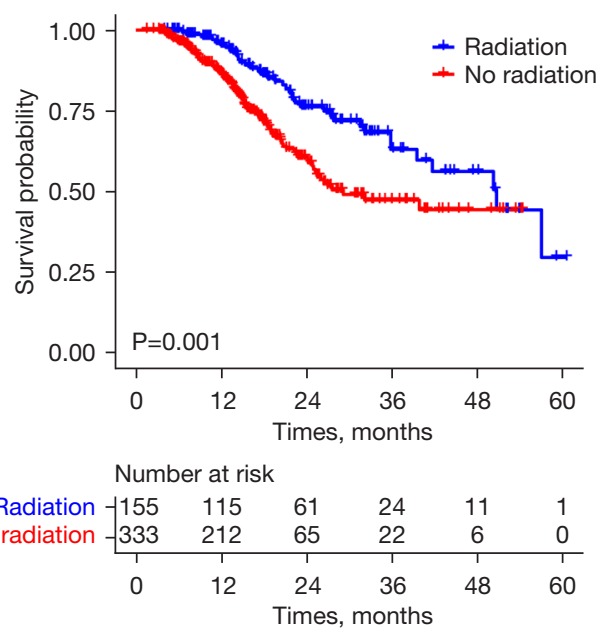

B

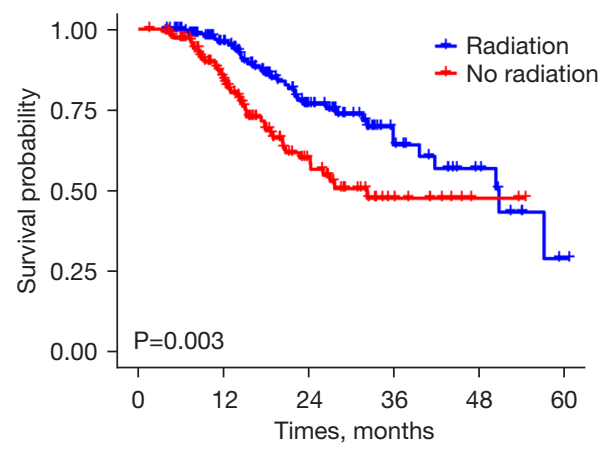

Number at risk

No radiation \begin{tabular}{cccccc}
-144 & 107 & 58 & 23 & 10 & 1 \\
-144 & 89 & 34 & 11 & 2 & 0 \\
\hline 0 & 12 & 24 & 36 & 48 & 60 \\
& & Times, months & &
\end{tabular}

Figure $1 \mathrm{KM}$ OS curves for RT and non-RT group patients before and after PSM. (A) KM curves for RT and non-RT group patients before PSM. (B) KM curves for RT and non-RT group patients after PSM. KM, Kaplan-Meier; OS, overall survival; RT, radiation therapy; PSM, propensity score matching.

results are shown in Table 3. They indicated that RT patients who received systemic therapy before RT had a significantly better mortality rate [hazard ratio $(\mathrm{HR})=0.218 ; 95 \%$ confidence interval (CI): 0.078-0.604), $\mathrm{P}=0.003$ ]. Moreover, the survival of RT patients was significantly associated with the site of RT for liver and lungs (HR $=3.317 ; 95 \% \mathrm{CI}$ : 1.126-9.776, $\mathrm{P}=0.030)$.

\section{Discussion}

In this study, we systematically evaluated the association of palliative RT with the OS of mCRC patients. The results demonstrated that patients treated with RT had a lower risk of death than those who did not receive RT. Numerous prior studies have addressed the role RT plays in treating 
Table 2 Univariate and multivariate analyses of OS in the PSM group

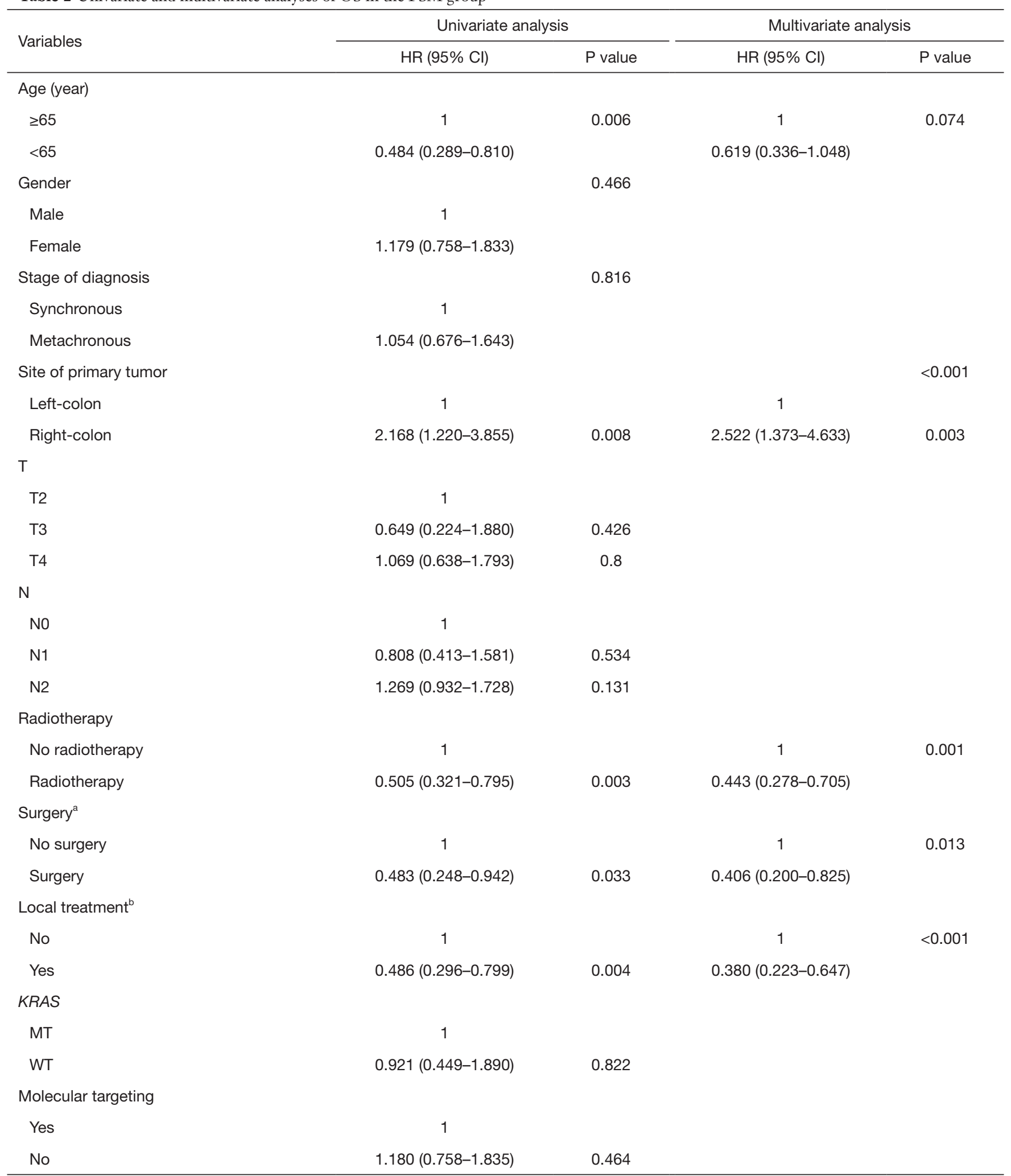

a , surgery of primary site; ${ }^{\mathrm{b}}$, local treatment of metastasis (including surgery, ablation, and intervention). OS, overall survival; PSM, propensity score matching; HR, hazard ratio; $\mathrm{Cl}$, confidence interval; MT, mutation; WT, wild-type. 
A
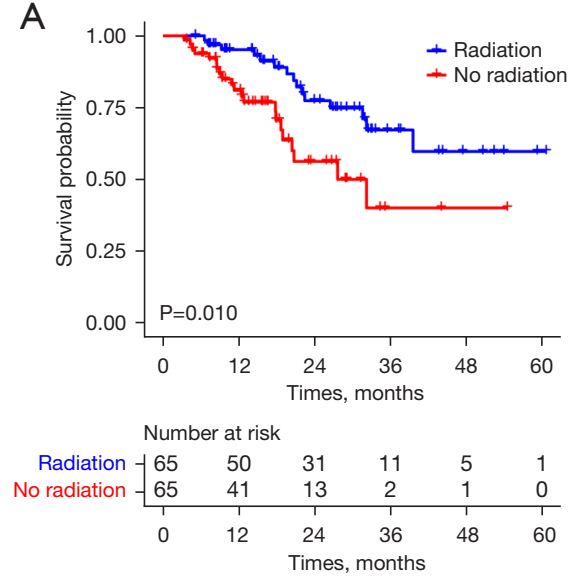

D

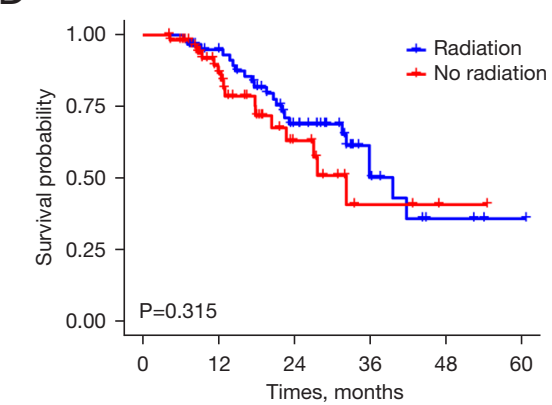

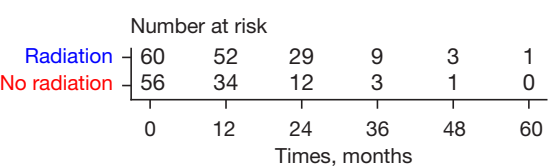

B
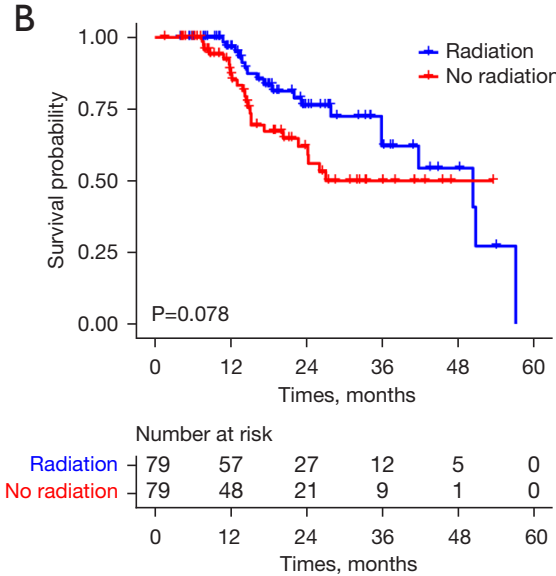

$\mathrm{E}$

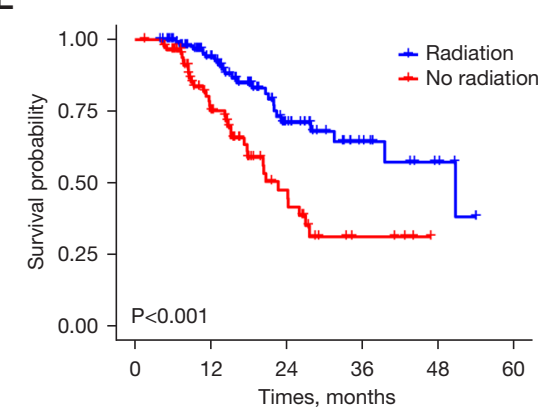

Number at risk

No radiation \begin{tabular}{cccccc}
-101 & 69 & 31 & 13 & 5 & 0 \\
-85 & 45 & 16 & 4 & 0 & 0 \\
\hline 1 & 1 & 1 & 1 & 1 & 1 \\
0 & 12 & 24 & 36 & 48 & 60 \\
& & & &
\end{tabular}

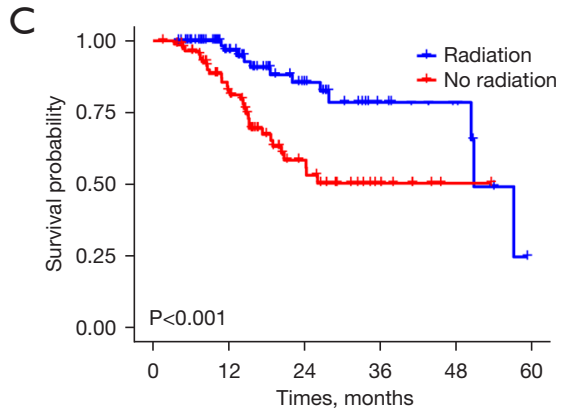

Number at risk

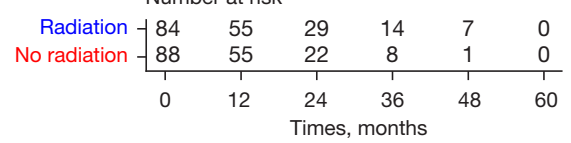

$\mathrm{F}$
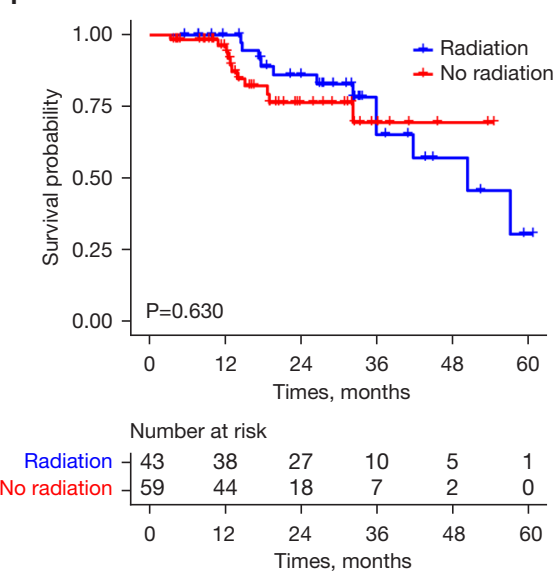

Figure 2 Subgroup survival analysis for RT and non-RT group patients after PSM. (A) KM analysis of OS in simultaneous metastasis subgroup. (B) KM analysis of OS in metachronous metastasis subgroup. (C) KM analysis of OS in the subgroup of patients who did not receive targeted treatment. (D) KM analysis of OS in the subgroup of patients who received targeted treatment. (E) KM analysis of OS in the subgroup of patients who did not receive local treatment. (F) KM analysis of OS in the subgroup of patients who received local treatment. RT, radiation therapy; PSM, propensity score matching; KM, Kaplan-Meier; OS, overall survival.

mCRC patients. Some literatures regard the local tumor control rate as the main endpoint, so long-term survival data has not been reported. Kim et al. evaluated the effects of RT on the survival of patients with stage IV rectal cancer through a meta-analysis. In their study, patients who received pelvic RT (RT group), and patients who did not receive radiotherapy (non-RT group), were paired using propensity scores to compare their survival prognosis. The findings suggested that the LR-free survival (LRFS) rate in the RT group was significantly higher than that in the non-RT group (2-year LRFS: $100 \%$ vs. $83.6 \%, \mathrm{P}=0.038$ ). At the same time, the results of their meta-analysis showed that the risk of LR was lower in the RT group than in the
non-RT group (17). From this, pelvic RT may be seen as beneficial to the local control (LC) of patients with stage IV rectal cancer, however, no significant difference was observed in the OS between the two groups. In our present study, the RT group recorded a significant improvement in survival time when compared with the non-RT group (50.8 vs. 32.2 months; $\mathrm{P}=0.003$ ). In another study, Franzese et al. collected the medical records of a total of 270 CRC patients who underwent SBRT. In this cohort 437 metastatic tumors were treated. Lung metastasis accounted for $48.5 \%$, followed by liver (36.4\%), and lymph nodes (12.4\%). In addition, 199 patients (73.7\%) underwent systemic treatment before SBRT treatment. The median 
Table 3 Univariate and multivariate analyses of survival in patients treated with RT

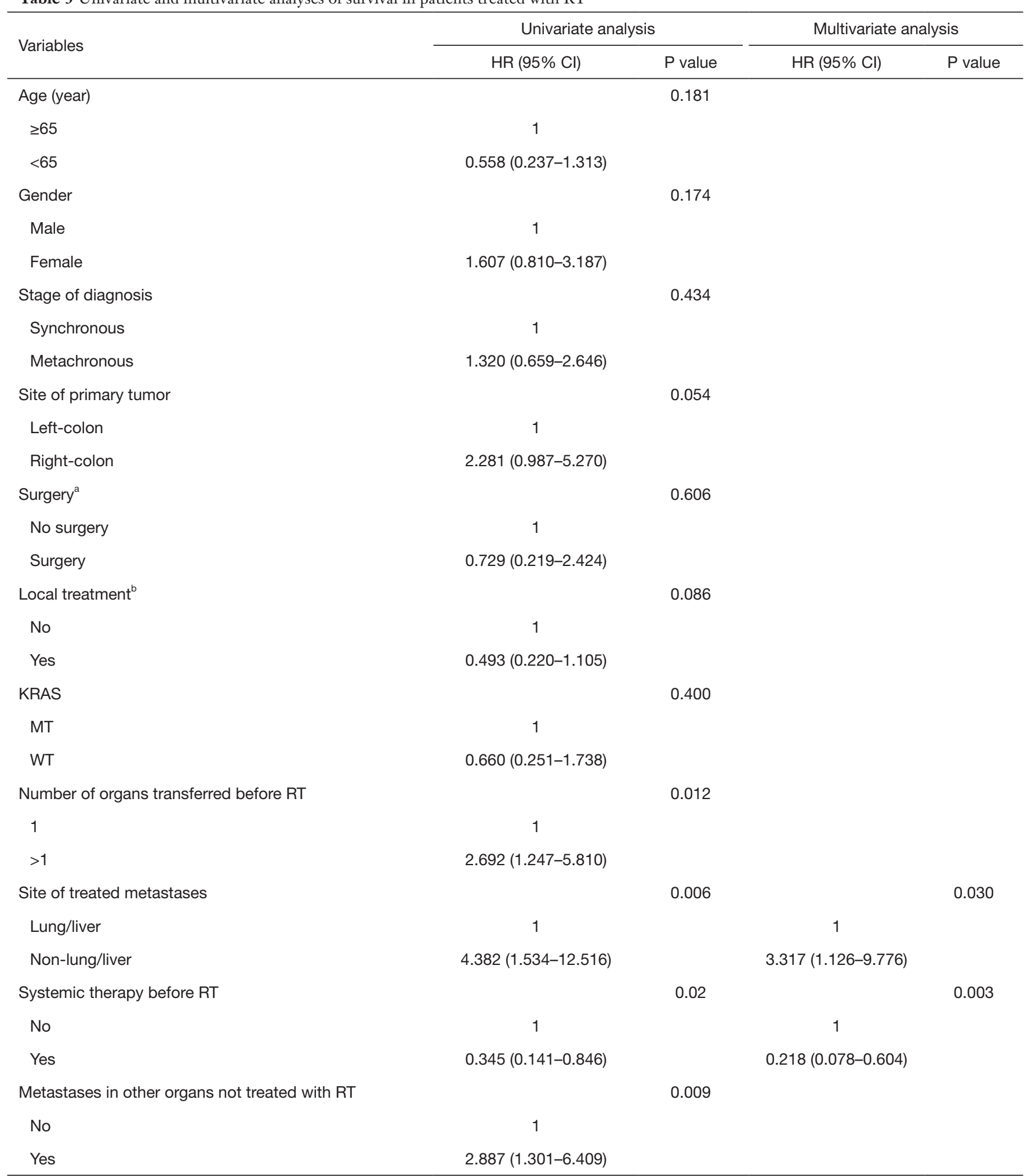

${ }^{a}$, surgery of primary site; ${ }^{\mathrm{b}}$, local treatment of metastasis (including surgery, ablation, and intervention). RT, radiation therapy; HR, hazard ratio; $\mathrm{Cl}$, confidence interval; MT, mutation; WT, wild-type. 
follow-up time was 23 months. After receiving SBRT, the LC rate at 1-, 3-, and 5-year intervals was $95 \%, 73 \%$, and $73 \%$, respectively, while the OS rate at 1-, 3-, and 5-year intervals was $88.5 \%, 56.6 \%$, and $37.2 \%$, respectively (18). Other evidence that supports our research includes a retrospective study by Liu et al., in which they found that palliative RT in patients with advanced rectal cancer improved OS. A subgroup analysis of all the patients who underwent surgery in their study showed that the 5-year survival rate of the RT and non-RT groups was $30.3 \%$ and $18.0 \%$, respectively (19). However, it is important to note that the above study only included patients with advanced rectal cancer, while our study also included colon cancer patients. The 1- and 2-year OS rates of the RT group in our study were $73.6 \%$ and $40.3 \%$, respectively, a finding consistent with those reported in the above literatures. In addition, the 1- and 2-year OS rates in this study were lower than the data reported in the other literatures. This discrepancy could be attributed to the metastasis status of the patients and the RT technique used in our study. In our research, patients treated with SBRT were not analyzed separately, and the RT techniques also included conventional RT, as well as IMRT. Moreover, when compared with the previous studies on oligometastasis, the patients in our study usually had multi-organ metastasis and multi-lesion metastasis.

Invasion and metastasis are important characteristics of malignant tumors. It has been reported that nearly $20 \%$ of patients with CRC were also diagnosed with metastasis. Several studies have reported that simultaneous liver metastasis is associated with poor outcomes of CRC patients $(20,21)$. Slesser et al. conducted a meta-analysis of the molecular markers related to colorectal liver metastasis in synchronous and metachronous groups. They found that patients with simultaneous liver metastasis had more aggressive molecular markers, such as TGF- $\beta$, COX-2, etc. (22). Moreover, a meta-analysis published by Tan et al. analyzed 21 articles to compare the survival rates of patients with simultaneous or metachronous liver metastasis after surgery. Results showed a reduction of $8-16 \%$ in the 5 -year survival of the simultaneous metastasis group (23). In our study, metachronous metastasis was defined if the time interval between the occurrence of metastasis and the initial diagnosis was more than 6 months. However, the results of our subgroup analysis showed that the OS was significantly improved in patients with simultaneous metastasis $(\mathrm{P}=0.010)$. It could therefore be assumed that RT can significantly improve the survival of patients with simultaneous metastasis.
The results of our study can be seen to enhance the findings of previous studies, as it also found patient survival improved in the simultaneous subgroup. A possible explanation for this could be that because of the poor prognosis of these patients the intervention of RT played a more significant role. In our study, the local treatment of metastasis was defined as other local treatments besides RT, including surgery, ablation, and intervention. Local treatment of metastasis is known to be one of the most useful therapeutic options in the treatment of mCRC patients (24-29). Nevertheless, surgery is not suitable for elderly patients who have large lesions close to blood vessels, or cardiopulmonary dysfunction. Radiofrequency ablation has a limited effect on lesions which have a diameter of $>3 \mathrm{~cm}$ and are near to blood vessels, bile ducts, or intestines. Interestingly, our research found that for patients who did not receive local treatment (surgery/ ablation/intervention), there was a significant improvement of OS with RT $(\mathrm{P}<0.001)$. In this subgroup, we found RT intervention could be an important supplementary method for local treatment, and may increase the OS. Moreover, there were also differences in the subgroup analysis of patients who did not receive targeted therapy. The results showed that the median OS of the RT group was 50.8 months, whereas a median OS was not achieved in the non-RT group $(\mathrm{P}<0.001)$. Although radiotherapy could improve the survival of the targeted therapy subgroup, no significant difference in the effect of RT between the groups could be statistically confirmed. It seems possible that these results could be attributed to a larger baseline risk in the groups that did not receive targeted therapy. From this, RT's absolute risk reduction of death was probably smaller among patients with targeted therapy.

Through both univariate and multivariate analyses, leftsided colon and rectal cancer, surgery of primary site, local treatment of metastasis, as well as RT were the independent prognostic factors. Subgroup analysis by the status of metastasis, targeted therapy, and other confounding factors further confirmed the therapeutic effects of palliative RT combined systemic with treatment. These results align with previous studies (30,31). For example, Liu et al. also found that patient age at diagnosis, patient marital status at diagnosis, tumor grade, $\mathrm{T}$ stage, $\mathrm{N}$ stage, tumor histology, patient surgical status, and tumor size were independent factors affecting the prognosis of patients with advanced rectal cancer who underwent palliative RT (19). Tsai et al. retrospectively analyzed the data from 155 patients with $\mathrm{mCRC}$ and concluded that simultaneous metastasis and late-stage primary tumors were independent factors 
affecting prognosis (32).

There are some limitations that should be recognized in our study. Firstly, our study is retrospective, and the case data we collected were from a single hospital in China, which compromises the generalizability of our results. In addition, some missing information due to uncontrollable factors (such as short follow-up times), also affected the credibility of the results. It is hoped that our study might enrich the RT database of $\mathrm{mCRC}$ and provide further evidence for using RT in the treatment of mCRC in the future. Currently, there are few clinical reports about immunotherapy combined with radiotherapy in CRC. Radiotherapy combined with immunotherapy is a new direction in the treatment of advanced CRC, which may bring survival benefits. We look forward to conducting further randomized controlled studies to provide more evidence for the resolution of this clinical problem.

\section{Conclusions}

Taken together, our study suggested that systemic therapy combined with palliative RT could significantly improve the long-term survival of patients with $\mathrm{mCRC}$, and that palliative RT was an independent prognostic factor. Subgroup analysis demonstrated a favorable OS outcome for patients with simultaneous metastasis and for patients who did not receive targeted therapy. In addition, the subgroup analyses, which were performed according to whether patients received local treatment (including surgery, ablation, and intervention) also produced similar results. The findings reported here shed new light on the application of using palliative RT to treat mCRC. In addition, we note that more work needs to be done to provide more definitive evidence.

\section{Acknowledgments}

Funding: This work was supported by the National Natural Science Foundation of China (Grant No. 81874084 to JR).

\section{Footnote}

Reporting Checklist: The authors have completed the STROBE reporting checklist. Available at https://dx.doi. org/10.21037/jgo-21-540

Data Sharing Statement: Available at https://dx.doi. org/10.21037/jgo-21-540
Conflicts of Interest: All authors have completed the ICMJE uniform disclosure form (available at https://dx.doi. org/10.21037/jgo-21-540). The authors have no conflicts of interest to declare.

Ethical Statement: The authors are accountable for all aspects of the work in ensuring that questions related to the accuracy or integrity of any part of the work are appropriately investigated and resolved. All procedures performed in this study involving human participants were in accordance with the Declaration of Helsinki (as revised in 2013). The study was approved by the ethics committee of the Wuhan Union Hospital. Because of the retrospective nature of the research, the requirement for informed consent was waived. The raw data are available from the corresponding author upon reasonable request and with the permission of Institutional Review Board of the Wuhan Union Hospital.

Open Access Statement: This is an Open Access article distributed in accordance with the Creative Commons Attribution-NonCommercial-NoDerivs 4.0 International License (CC BY-NC-ND 4.0), which permits the noncommercial replication and distribution of the article with the strict proviso that no changes or edits are made and the original work is properly cited (including links to both the formal publication through the relevant DOI and the license). See: https://creativecommons.org/licenses/by-nc-nd/4.0/.

\section{References}

1. Siegel RL, Miller KD, Goding Sauer A, et al. Colorectal cancer statistics, 2020. CA Cancer J Clin 2020;70:145-64.

2. Sharma A, Baker S, Duijm M, et al. Prognostic factors for local control and survival for inoperable pulmonary colorectal oligometastases treated with stereotactic body radiotherapy. Radiother Oncol 2020;144:23-9.

3. Sánchez-Gundín J, Fernández-Carballido AM, MartínezValdivieso L, et al. New trends in the therapeutic approach to metastatic colorectal cancer. Int J Med Sci 2018;15:659-65.

4. de Gramont A, Bosset JF, Milan C, et al. Randomized trial comparing monthly low-dose leucovorin and fluorouracil bolus with bimonthly high-dose leucovorin and fluorouracil bolus plus continuous infusion for advanced colorectal cancer: a French intergroup study. J Clin Oncol 1997;15:808-15. 
5. Saltz LB, Cox JV, Blanke C, et al. Irinotecan plus fluorouracil and leucovorin for metastatic colorectal cancer. Irinotecan Study Group. N Engl J Med 2000;343:905-14.

6. Loree JM, Kopetz S. Recent developments in the treatment of metastatic colorectal cancer. Ther Adv Med Oncol 2017;9:551-64.

7. Ly BH, Vlastos G, Rapiti E, et al. Local-regional radiotherapy and surgery is associated with a significant survival advantage in metastatic breast cancer patients. Tumori 2010;96:947-54.

8. Hu J, Kong L, Gao J, et al. Use of radiation therapy in metastatic nasopharyngeal cancer improves survival: a SEER analysis. Sci Rep 2017;7:721.

9. Shao YH, Kim S, Moore DF, et al. Cancer-specific survival after metastasis following primary radical prostatectomy compared with radiation therapy in prostate cancer patients: results of a population-based, propensity scorematched analysis. Eur Urol 2014;65:693-700.

10. Gomez DR, Tang C, Zhang J, et al. Local consolidative therapy vs. maintenance therapy or observation for patients with oligometastatic non-small-cell lung cancer: long-term results of a multi-institutional, phase II, randomized study. J Clin Oncol 2019;37:1558-65.

11. Folkesson J, Birgisson H, Pahlman L, et al. Swedish Rectal Cancer Trial: long lasting benefits from radiotherapy on survival and local recurrence rate. J Clin Oncol 2005;23:5644-50.

12. Park JH, Yoon SM, Yu CS, et al. Randomized phase 3 trial comparing preoperative and postoperative chemoradiotherapy with capecitabine for locally advanced rectal cancer. Cancer 2011;117:3703-12.

13. Ngan SY, Burmeister B, Fisher RJ, et al. Randomized trial of short-course radiotherapy versus long-course chemoradiation comparing rates of local recurrence in patients with $\mathrm{T} 3$ rectal cancer: Trans-Tasman Radiation Oncology Group trial 01.04. J Clin Oncol 2012;30:3827-33.

14. Thomas PR, Lindblad AS. Adjuvant postoperative radiotherapy and chemotherapy in rectal carcinoma: a review of the Gastrointestinal Tumor Study Group experience. Radiother Oncol 1988;13:245-52.

15. Austin PC. Optimal caliper widths for propensity-score matching when estimating differences in means and differences in proportions in observational studies. Pharm Stat 2011;10:150-61.

16. Yao XI, Wang X, Speicher PJ, et al. Reporting and guidelines in propensity score analysis: a systematic review of cancer and cancer surgical studies. J Natl Cancer Inst 2017;109:djw323.
17. Kim MJ, Kim SJ, Park SC, et al. Adjuvant radiotherapy for the treatment of stage $I V$ rectal cancer after curative resection: a propensity score-matched analysis and metaanalysis. Medicine (Baltimore) 2016;95:e4925.

18. Franzese C, Comito T, Toska E, et al. Predictive factors for survival of oligometastatic colorectal cancer treated with stereotactic body radiation therapy. Radiother Oncol 2019;133:220-6.

19. Liu Q, Shan Z, Luo D, et al. Palliative beam radiotherapy offered real-world survival benefit to metastatic rectal cancer: a large US population-based and propensity scorematched study. J Cancer 2019;10:1216-25.

20. Adam R, de Gramont A, Figueras J, et al. Managing synchronous liver metastases from colorectal cancer: a multidisciplinary international consensus. Cancer Treat Rev 2015;41:729-41.

21. Ekberg H, Tranberg KG, Andersson R, et al. Determinants of survival in liver resection for colorectal secondaries. Br J Surg 1986;73:727-31.

22. Slesser AA, Georgiou P, Brown G, et al. The tumour biology of synchronous and metachronous colorectal liver metastases: a systematic review. Clin Exp Metastasis 2013;30:457-70.

23. Tan EK, Ooi LL. Colorectal cancer liver metastases understanding the differences in the management of synchronous and metachronous disease. Ann Acad Med Singap 2010;39:719-15.

24. Kopetz S, Chang GJ, Overman MJ, et al. Improved survival in metastatic colorectal cancer is associated with adoption of hepatic resection and improved chemotherapy. J Clin Oncol 2009;27:3677-83.

25. Choti MA, Sitzmann JV, Tiburi MF, et al. Trends in long-term survival following liver resection for hepatic colorectal metastases. Ann Surg 2002;235:759-66.

26. Mise Y, Kopetz S, Mehran RJ, et al. Is complete liver resection without resection of synchronous lung metastases justified? Ann Surg Oncol 2015;22:1585-92.

27. Rhim H, Goldberg SN, Dodd GD 3rd, et al. Essential techniques for successful radio-frequency thermal ablation of malignant hepatic tumors. Radiographics 2001;21 Spec No:S17-35; discussion S36-9.

28. Hasegawa T, Takaki H, Kodama H, et al. Three-year survival rate after radiofrequency ablation for surgically resectable colorectal lung metastases: a prospective multicenter study. Radiology 2020;294:686-95.

29. Gruber-Rouh T, Marko C, Thalhammer A, et al. Current strategies in interventional oncology of colorectal liver metastases. Br J Radiol 2016;89:20151060. 
30. Lee JH, Jo IY, Lee JH, et al. The role of postoperative pelvic radiation in stage IV rectal cancer after resection of primary tumor. Radiat Oncol J 2012;30:205-12.

31. Kuo IM, Huang SF, Chiang JM, et al. Clinical features and prognosis in hepatectomy for colorectal cancer with centrally located liver metastasis. World J Surg Oncol 2015;13:92.

Cite this article as: Ba L, Wang Q, Wang H, Zhu L, Zhang T, Ren J, Lin Z. Survival analysis and prognostic factors of palliative radiotherapy in patients with metastatic colorectal cancer: a propensity score analysis. J Gastrointest Oncol 2021;12(5):2211-2222. doi: 10.21037/jgo-21-540
32. Tsai MS, Su YH, Ho MC, et al. Clinicopathological features and prognosis in resectable synchronous and metachronous colorectal liver metastasis. Ann Surg Oncol 2007;14:786-94.

(English Language Editor: J. Goetz) 